International Conference on Ceramics, Bikaner, India

International Journal of Modern Physics: Conference Series

Vol. 22 (2013) 576-582

(C) World Scientific Publishing Company

DOI: $10.1142 / \mathrm{S} 2010194513010696$

\title{
A BRIEF SURVEY ON BASIC PROPERTIES OF THIN FILMS FOR DEVICE APPLICATION
}

\author{
M. C. RAO \\ Department of Physics, Andhra Loyola College \\ Vijayawada - 520008, India \\ raomc72@gmail.com \\ M. S. SHEKHAWAT \\ Department of Physics, Govt. Engineering College Bikaner \\ Bikaner - 334004, India \\ manoj.shekhawat1@gmail.com
}

\begin{abstract}
Thin film materials are the key elements of continued technological advances made in the fields of optoelectronic, photonic and magnetic devices. Thin film studies have directly or indirectly advanced many new areas of research in solid state physics and chemistry which are based on phenomena uniquely characteristic of the thickness, geometry and structure of the film. The processing of materials into thin films allows easy integration into various types of devices. Thin films are extremely thermally stable and reasonably hard, but they are fragile. On the other hand organic materials have reasonable thermal stability and are tough, but are soft. Thin film mechanical properties can be measured by tensile testing of freestanding films and by the micro beam cantilever deflection technique, but the easiest way is by means of nanoindentation. Optical experiments provide a good way of examining the properties of semiconductors. Particularly measuring the absorption coefficient for various energies gives information about the band gaps of the material. Thin film materials have been used in semiconductor devices, wireless communications, telecommunications, integrated circuits, rectifiers, transistors, solar cells, lightemitting diodes, photoconductors and light crystal displays, lithography, micro- electromechanical systems (MEMS) and multifunctional emerging coatings, as well as other emerging cutting technologies.
\end{abstract}

Keywords: Thin films; Optical properties; Mechanical properties; electrical properties; Applications.

\section{Introduction}

The field of material science and engineering community's ability to conceive the novel materials with extraordinary combination of chemical, physical and mechanical properties has changed the modern society. There is an increasing technological progress. Modern technology requires thin films for different applications. Thin film technology is the basic of astounding development in solid state electronics. The usefulness of the optical properties of metal films and scientific curiosity about the behavior of twodimensional solids has been responsible for the immense interest in the study science and 
technology of the thin films. Thin film studies have directly or indirectly advanced many new areas of research in solid state physics and chemistry which are based on phenomena uniquely characteristic of the thickness, geometry and structure of the film ${ }^{1}$. The phenomenal rise in thin film researches is no doubt due to their extensive applications in the diverse fields of electronics, optics, space science, aircrafts, defense and other industries. These investigations have led a numerous inventions in the forms of active devices and passive components, piezo-electric devices, micro-miniaturization of power supply, rectification and amplification, sensor elements, storage of solar energy and its conversion to other form, magnetic memories, super conduction films, interference filters, reflecting and antireflection coatings and many others ${ }^{2}$.

Thin film materials are the key elements of continued technological advances made in the fields of optoelectronic, photonic and magnetic devices. The processing of materials into thin films allows easy integration into various types of devices. The properties of material significantly differ when analyzed in the form of thin films. Most of the functional materials are rather applied in thin film form due to their specific electrical, magnetic, optical properties or wear resistance. Thin film technologies make use of the fact that the properties can particularly be controlled by the thickness parameter. Thin films are formed mostly by deposition, either physical or chemical methods. Thin films, both crystalline and amorphous, have immense importance in the age of high technology. Few of them are: microelectronic devices, magnetic thin films in recording devices, magnetic sensors, gas sensor, A. R. coating, photoconductors, IR detectors, interference filters, solar cells, polarizer's, temperature controller in satellite, superconducting films, anticorrosive and decorative coatings ${ }^{3}$.

Nano-sculptured thin films are a new class of films deposited on substrates with controlled azimuthal rotation, $\omega$ and tilt, $\theta$, by a method called glancing angle deposition (GLAD). The understanding and modeling of nano-sculptured films deposited on tilted substrates become increasingly important as their applications encompass various disciplines: photonics liquid crystal display technology, magnetic media information storage, organic or inorganic sensors, energy storage technology, among others. Thickness calibration is a common problem encountered in the case of these films primarily as a result of substrate tilt angle dependent porosity and flux capture ${ }^{4,5}$.

Thin films are extremely thermally stable and reasonably hard, but they are fragile. On the other hand organic materials have reasonable thermal stability and are tough, but are soft. For mechanical device stability four material properties of the device components are important: elastic modulus, yield strength, interfacial adhesion and film fracture toughness. Mechanical properties of thin films often differ from those of the bulk materials. This can be partially explained by the nanostructure of thin films and the fact that these films are attached to a substrate. Due to typically high yield strengths, thin films can support very high residual stresses. This residual stress can be relieved later during processing or in the actual device operation through plastic deformation, thin film fracture, or interfacial delamitation. Both elastic and plastic properties are important for thin film characterization. Thin film mechanical properties can be measured by tensile 
testing of freestanding films and by the micro beam cantilever deflection technique, but the easiest way is by means of nanoindentation, since no special sample preparation is required and tests can be performed quickly and inexpensively. Nanoindentation is a versatile technique for measuring films mechanical properties. During the measurement a sharp diamond indenter is forced into the tested material while continuously recording both the force and the indentation depth ${ }^{6}$.

\section{Properties}

\subsection{Optical properties}

Optical experiments provide a good way of examining the properties of semiconductors. Particularly measuring the absorption coefficient for various energies gives information about the band gaps of the material. Knowledge of these band gaps is extremely important for understanding the electrical properties of a semiconductor and is therefore of great practical interest ${ }^{7}$.

\subsection{Mechanical properties}

Mechanical properties of thin films often differ from those of the bulk materials. This can be partially explained by the nanostructure of thin films and the fact that these films are attached to a substrate. Due to typically high yield strengths, thin films can support very high residual stresses. This residual stress can be relieved later during processing or in the actual device operation through plastic deformation, thin film fracture, or interfacial delamination $^{8}$. Both elastic and plastic properties are important for thin film characterization. Thin film mechanical properties can be measured by tensile testing of freestanding films and by the micro beam cantilever deflection technique, but the easiest way is by means of nanoindentation, since no special sample preparation is required and tests can be performed quickly and inexpensively ${ }^{9-11}$.

\subsection{Electrical properties}

There are three types of electron materials: metal, semiconductor and dielectric. Obviously, the electrical transport nature is different in all types of materials, therefore it is impossible describe all together. However, in the thin film form, scaling effects begin influence on the electrical properties of materials by the same way. The film thickness, the lattice dimensions, the purity, the surface roughness and the imperfect level of the layer are must significant parameters define the type, mechanism and stability of the electrical transport ${ }^{12}$. Fig. 1 shows the conductivity scale for various materials at room temperature. 


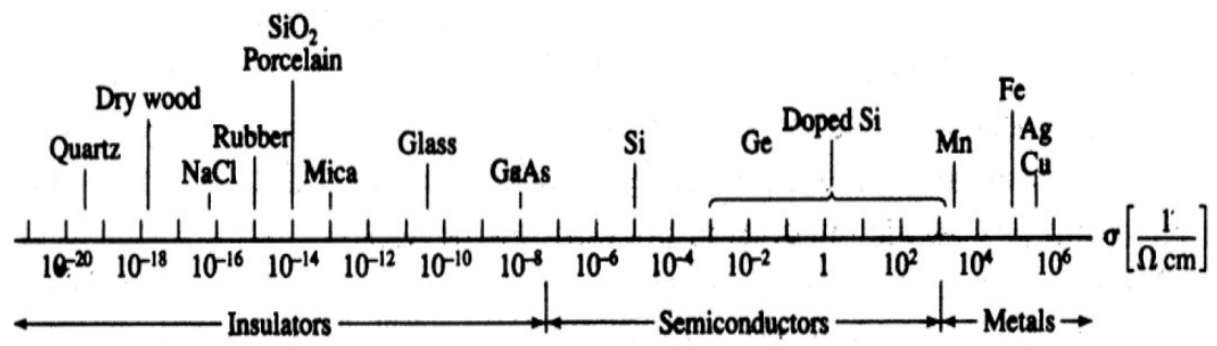

Fig. 1 Conductivity scale for various materials at room temperature

\section{Applications of thin films}

Although the study of thin film phenomena dates back well over a century, it is really only over the last four decades that they have been used to a significant extent in practical situations. The requirement of micro miniaturization made the use of thin and thick films virtually imperative. The development of computer technology led to a requirement for very high density storage techniques and it is this which has stimulated most of the research on the magnetic properties of thin films. Many thin film devices have been developed which have found themselves looking for an application. In general these devices have resulted from research into the physical properties of thin films.

Thin film materials have already been used in semiconductor devices, wireless communications, telecommunications, integrated circuits, rectifiers, transistors, solar cells, light-emitting diodes, photoconductors, light crystal displays, magneto-optic memories, audio and video systems, compact discs, electro-optic coatings, memories, multilayer capacitors, flat-panel displays, smart windows, computer chips, magneto optic discs, lithography, micro- electromechanical systems and multifunctional emerging coatings, as well as other emerging cutting technologies ${ }^{13}$.

\subsection{Data storage}

As the data storage density in cutting edge microelectronic devices continues to increase, the super paramagnetic effect poses a problem for magnetic data storage media. One strategy for overcoming this obstacle is the use of thermo mechanical data storage technology. In this approach, data is written by a nanoscale mechanical probe as an indentation on a surface, read by a transducer built into the probe, and then erased by the application of heat. An example of such a device is the IBM millipede, which uses a polymer thin film as the data storage medium. It is also possible; however, to use other kinds of media for thermo mechanical data storage and in the following work, we explore the possibility of using thin film Ni-Ti shape memory alloy (SMA). Previous work has shown that nanometer-scale indentations made in marten site phase Ni-Ti SMA thin films recover substantially upon heating. Issues such as repeated thermo mechanical cycling of indentations indent proximity and film thickness impact the practicability of this technique. While there are still problems to be solved, the experimental evidence and 
theoretical predictions show SMA thin films are an appropriate medium for thermo mechanical data storage ${ }^{14}$.

\subsection{Flat panel displays}

The Flat Panel Display (FPD) fabrication environment is among the worlds most competitive and technologically complex. Device designers and manufacturers continually strive to satisfy the worldwide consumer's appetite for larger displays, greater pixel resolution and feature-rich performance, all at a lower cost than the previous generation of technology. The need to control contamination in air, gas and liquid process streams is now a paramount focus of process engineers and designers ${ }^{15}$.

\subsection{Optoelectronic}

An optoelectronic thin-film chip, comprising at least one radiation-emitting region in an active zone of a thin-film layer and a lens disposed downstream of the radiation emitting region, said lens being formed by at least one partial region of the thin-film layer, the lateral extent of the lens being greater than the lateral extent of the radiation emitting region. The thin-film layer is provided for example by a layer sequence which is deposited epitaxially on a growth substrate and from which the growth substrate is at least partly removed. That is to say that the thickness of the substrate is reduced. In other words, the substrate is thinned. It is furthermore possible for the entire growth substrate to be removed from the thin-film layer. The thin-film layer has at least one active zone suitable for generating electromagnetic radiation. The active zone may be provided for example by a layer or layer sequence which has a p-n junction, a double heterostructure, a single quantum well structure or a multiple quantum well structure. Particularly preferably, the active zone has at least one radiation-emitting region. In this case, the radiation-emitting region is formed for example by a partial region of the active zone. Electromagnetic radiation is generated in said partial region of the active zone during operation of the optoelectronic thin-film chip ${ }^{16}$.

\subsection{Optical coatings}

An optical coating is one or more thin layers of material deposited on an optical component such as a lens or mirror, which alters the way in which the optic reflects and transmits light. One type of optical coating is an antireflection coating, which reduces unwanted reflections from surfaces, and is commonly used on spectacle and photographic lenses. Another type is the high-reflector coating which can be used to produce mirrors which reflect greater than $99.99 \%$ of the light which falls on them. More complex optical coatings exhibit high reflection over some range of wavelengths, and anti-reflection over another range, allowing the production of dichroic thin-film optical filters ${ }^{17}$. 


\subsection{Thin film lithium ion batteries}

They are similar to lithium-ion batteries, but they are composed of thin materials, some only nanometers or micrometers thick, which allow for the finished battery to be just millimeters thick. They have been developed and advanced primarily within the last decade. These batteries consist of a substrate, electrolyte, current collector, anode, cathode and a charge separator. There has been much research into the determination of the most effective components for this type of battery. It has been shown recently that even ordinary printer paper can be used as a charge separator and a substrate. These thin film batteries are an improvement on the common secondary, or rechargeable, lithium ion batteries in many ways. These batteries exhibit the same voltage and current as their bulky counterparts, but their thinner dimensions allow for greater applications such as making thinner electronic devices, like cell phones and laptops and even implantable medical devices and reducing the weight of common devices that are run on battery power because of the batteries' high energy density. These batteries can be formed into any shape and they can be stacked, to be used in parallel, thus even further reducing the space needed for a battery. Fig. 2 shows the schematic representation of thin film lithium ion batteries ${ }^{18,19}$.

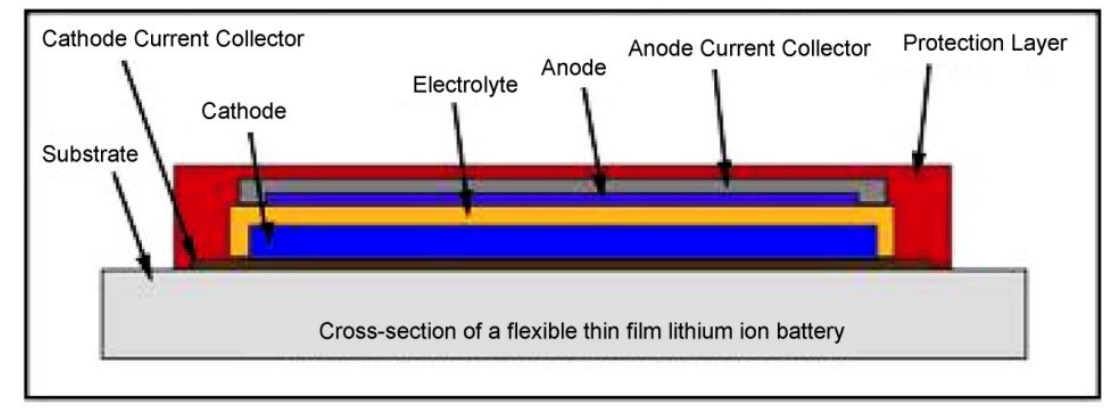

Fig. 2 Schematic representation of thin film lithium ion batteries

\section{Conclusions}

Thin film technology is the basic of astounding development in solid state electronics. The usefulness of the optical properties of metal films, and scientific curiosity about the behavior of two-dimensional solids has been responsible for the immense interest in the study science and technology of the thin films. Due to typically high yield strengths, thin films can support very high residual stresses. This residual stress can be relieved later during processing or in the actual device operation through plastic deformation, thin film fracture, or interfacial delamination. Mechanical properties of thin films often differ from those of the bulk materials. This can be partially explained by the nanostructure of thin films and the fact that these films are attached to a substrate. There are three types of electron materials: metal, semiconductor and dielectric. Obviously, the electrical transport nature is different in all types of materials, therefore it is impossible describe all 
together. Thin film materials have already been used in semiconductor devices, multilayer capacitors, flat-panel displays, smart windows, computer chips, magneto optic discs, lithography and micro-electromechanical systems.

\section{Acknowledgments}

The author (M. C. Rao) is thankful to UGC for providing the financial assistance through Major Research Project (Link No. F. No. 40-24/2011(SR))

\section{References}

1. A.R. West, 'Solid State Chemistry' John willey \& Sons, Singapore (2003).

2. B. K. Kuanr, S. Maat, S. Chandrashekariaih, V. Veerakumar, R. E. Camley and Z. Celinski, J. Appl. Phys. 103, 07C107 (2008).

3. Y. Gao, H. Niu and C. Q. Chen, Chem. Phys. Lett. 367, 141 (2003).

4. K. Robbie, G. Beydaghyan, T. Brown, C. Dean, J. Adams and C. Buzea, Rev. Sci. Instrum. 75, 1089 (2004).

5. K. Robbie, M. J. Brett and A. Lakhtakia, J. Vac. Sci. Technol. A 13, 2991 (1995).

6. C. Fischer-Cripps, Nanoindentation, New York, Springer, (2002).

7. J. Fraxedas, Adv. Mater. 14, 1603 (2002).

8. D. T. Read, J. W. Dally, J. Mater. Res. 8, 1542 (1993).

9. T. P. Weihs, S. Hong, J. C. Bravman and W. D. Nix, J. Mater. Res. 3, 931 (1998).

10. S. P. Baker and W. D. Nix, J. Mater. Res. 9, 3131 (1994).

11. S. P. Baker and W. D. Nix, J. Mater. Res. 9, 3145 (1994).

12. M.I. Alonso, M. Garriga, J.O. Oss, F. Schreiber, E. Barrena and H. Dosch, J. Chem. Phys. 119, 6335 (2003).

13. J. Dubowik, K. Zaleski, H. G lowinski and I. Goscianska, Phys. Rev. B 84, 184438 (2011).

14. S. S. Kalarickal, P. Krivosik, M. Wu, C. E. Patton, M. L. Schneider, P. Kabos, T. J. Silva and J. P. Nibarger, J. Appl. Phys. 99, 093909 (2006).

15. O. Mosendz, V. Vlaminck, J. E. Pearson, F. Y. Fradin, G. E. W. Bauer, S. D. Bader and A. Hoffmann, Phys. Rev. B 82, 214403 (2010).

16. C. Bilzer, T. Devolder, P. Crozat, C. Chappert, S. Cardoso and P. P. Freitas, J. Appl. Phys. 101, 074505 (2007).

17. J. Nogues and I. K. Schuller, J. Magn. Magn. Mater. 192, 203 (1999).

18. R. D. McMichael, M. D. Stiles, P. J. Chen, and W. F. Egelhoff, Jr., Phys. Rev. B 58, 8605 (1998).

19. M. D. Stiles and R. D. McMichael, Phys. Rev. B 59, 3722 (1999). 